\title{
Twenty years of diabetes surveillance using the Canadian Chronic Disease Surveillance System
}

\author{
Allana G. LeBlanc, PhD; Yong Jun Gao, MSc; Louise McRae, BSc; Catherine Pelletier, MSc
}

Tweet this article

\begin{abstract}
In 1999, the Government of Canada, along with the provinces and territories, established the National Diabetes Surveillance System (NDSS) to track rates of diabetes in Canada. The NDSS used a novel method to systematically collect and report national diabetes data using linked administrative health databases. The NDSS has since evolved to become the Canadian Chronic Disease Surveillance System (CCDSS) and provides information on over 20 chronic conditions. This At-a-glance report provides the most up-to-date CCDSS information on diabetes rates in Canada. Currently, 8.8\% of Canadians ( $9.4 \%$ male, $8.1 \%$ female, aged one year and older) live with diabetes, and approximately 549 new cases are diagnosed each day. Since 2000, the age-standardized prevalence rate has increased by an average of $3.3 \%$ per year. The age-standardized incidence rate has remained relatively stable, and all-cause mortality rates among those with diabetes have decreased by an average of $2.1 \%$ per year. This suggests that people are living longer with a diabetes diagnosis.
\end{abstract}

\section{Introduction}

Diabetes mellitus is a metabolic disorder characterized by impaired insulin secretion or action, resulting in hyperglycemia. Diabetes can result in a range of long-term complications such as cardiovascular disease, retinopathy, nephropathy, neuropathy, amputations and decreased life expectancy. ${ }^{1}$ The majority of diabetes cases can be classified into one of two categories: type 1 diabetes or type 2 diabetes. In the general population, it is estimated that approximately $90 \%$ of all diabetes diagnoses are type 2 diabetes, $9 \%$ are type 1 diabetes and $1 \%$ are other types of diabetes (e.g. gestational diabetes, other specific types related to gene or drug interactions). ${ }^{1}$ Type 1 diabetes is an autoimmune disease and cannot be prevented. ${ }^{1}$ Type 2 diabetes occurs due to a wide range of social, environmental and genetic factors. ${ }^{1,2}$ Risk factors for type 2 diabetes include obesity, unhealthy diet (e.g. high in ultra-processed foods), physical inactivity, lower socioeconomic status, increased age and ethnicity (e.g. increased risk among Black Canadians, South Asian Canadians, and Indigenous peoples)..$^{2,3}$ Data from the Canadian Health Measures Survey suggest approximately $7.3 \%$ of Canadians (aged 12 years and older) are living with diabetes. However, this is based on self-report data and may be an underestimation. ${ }^{4}$

To respond to the challenge of diabetes in Canada, the Government of Canada, along with provinces and territories, established the National Diabetes Surveillance System (NDSS) in 1999.5,6 Specifically, the NDSS was established to enable ongoing surveillance of diabetes and its complications; to create a national standardized database through the integration of new and existing databases; to disseminate national comparative information to inform effective prevention and treatment strategies for diabetes; and to provide a basis for evaluating economic or cost-related issues regarding the care, management and treatment of diabetes in Canada. ${ }^{5}$ Since it was launched, the NDSS has evolved to become
Highlights

- The Canadian Chronic Disease Surveillance System has provided important information on diabetes rates in Canada since 2000.

- Currently, $8.8 \%$ of Canadians $(9.4 \%$ male, $8.1 \%$ female, aged one year and older) live with diabetes.

- The age-standardized prevalence rate of diabetes has increased over time, whereas the age-standardized incidence rate has remained stable.

- The all-cause mortality rate among those with diabetes has decreased, suggesting people are living longer with a diabetes diagnosis.

the Canadian Chronic Disease Surveillance System (CCDSS), now under the responsibility of the Public Health Agency of Canada (PHAC), and is able to provide information on 20 chronic conditions. ${ }^{5}$ The CCDSS has been used previously to report on trends over time for chronic disease in Canada, including incidence, mortality and multimorbidity. ${ }^{7-10}$ The CCDSS first reported on diabetes in 2000; thus, 2019 marks 20 years of diabetes surveillance using the CCDSS. This work provides information on trends in diabetes prevalence, incidence and all-cause mortality in Canada since the inception of the CCDSS.

\section{Methods}

The Canadian Chronic Disease Surveillance System

Through the CCDSS, provincial and territorial health insurance registry records are 
linked using a unique personal identifier to the corresponding physician billing claims, hospital discharge abstract records and prescription drug records to provide information on incidence, prevalence and mortality. ${ }^{11-13}$ Data on identified disease cases are extracted by each province and territory using a standard analytical approach, based on case definitions for each CCDSS disease or condition. Individuallevel data are aggregated at the provincial or territorial level before submission to PHAC to protect patient privacy. Data are collected and updated on a regular basis and provide information by age group, sex, province/territory and trends over time. The CCDSS provides data for all Canadians who are eligible for provincial or territorial health insurance-approximately $97 \%$ of the population.

\section{Diabetes case definition}

Canadians aged 1 year and older are identified as having diagnosed diabetes if they have at least one hospitalization record, or at least two physician claims in a two-year period with an ICD (International Statistical Classification of Diseases and Related Health Problems) code for diabetes. The current case definition is unable to distinguish between type 1 and type 2 diabetes and reports on both types combined. Prevalence is lifetime prevalence and incidence is the first time the patient meets the criteria for diabetes. Valid ICD codes are: ICD-9-CM: 250, and ICD-10-CA: E10, E11, E12, E13, E14. To account for diabetes during pregnancy, records containing codes for diabetes are removed for women aged 10 to 54 years for 120 days preceding and 190 after hospital records containing any pregnancy or obstetrical code. Some provinces and territories have additional exclusions: data from Nova Scotia for individuals aged 1 to 19 are excluded; data from Yukon are excluded before 2010/11; data from Nunavut are excluded before 2005/06; data from Saskatchewan are not available for $2016 / 17$. Additional details on the diabetes case study can be found on the CCDSS online data tool (https:// health-infobase.canada.ca/ccdss/data-tool/) ${ }^{3}$ and elsewhere. ${ }^{5}$

\section{Statistical analysis}

CCDSS data presented here are current as of March 2019 and include Canadians aged 1 year and older with diagnosed type 1 and type 2 diabetes combined, excluding gestational diabetes. Data are presented for each fiscal year (i.e. April 1 to March 31). Crude rates were based on randomly rounded counts to an adjacent multiple of 10. Age-standardized rates are based on nonrounded counts and standardized to the 2011 final postcensal Canadian population released in 2013 using life-course age groups. Mortality rate ratio was calculated by dividing the all-cause mortality rate among individuals with diabetes by the all-cause mortality rate among individuals without diabetes. A rate ratio greater than one indicates that individuals with the disease experience a higher mortality burden compared to individuals without the disease, regardless of the cause of death. Assuming the baseline age-standardized mortality rates between those with and without the disease are similar, the difference in their all-cause mortality represented by the rate ratio can be attributed to deaths directly or indirectly related to the disease. Additional details can be found online in the CCDSS summary of methods (https://health-infobase.canada .ca/ccdss/data-tool/Methods).

We used Joinpoint software to run piecewise regression models to calculate change in age-standardized rates over time and identify any statistically significant changes in trends from 2000/01 to 2016/17 (Joinpoint software version 4.2.0.2, National Cancer Institute, Bethesda, MD, USA). This allowed us to identify time periods where the annual percent change differed significantly. The maximum number of joinpoints was set at four. The minimum number of observations from a joinpoint

\section{TABLE 1}

Diabetes rates in Canada, Canadian Chronic Disease Surveillance System, 2016/17

\begin{tabular}{|c|c|c|c|}
\hline Indicator & Total & Male & Female \\
\hline \multicolumn{4}{|l|}{ Prevalence $(\mathrm{n}, \%)$} \\
\hline Total population (aged $\geq 1$ year) & 3170969 (8.8) & $1686700(9.4)$ & $1484260(8.1)$ \\
\hline Children (aged 1-19 years) & $24330(0.3)$ & $12700(0.3)$ & $11630(0.3)$ \\
\hline Adults (aged $\geq 20$ years) & 3146630 (10.9) & $1674010(11.8)$ & $1472630(10.0)$ \\
\hline \multicolumn{4}{|l|}{ Incidence (n, rate per 100 000) } \\
\hline Total population (aged $\geq 1$ year) & $200400(603.5)$ & $109230(667.9)$ & $91160(540.9)$ \\
\hline Children (aged 1-19 years) & $3070(42.1)$ & $1530(40.9)$ & $1540(43.3)$ \\
\hline Adults (aged $\geq 20$ years) & $197330(761.5)$ & $107700(853.7)$ & $89630(674.0)$ \\
\hline $\begin{array}{l}\text { Mortality rate ratio (total } \\
\text { population, aged } \geq 1 \text { year; } \\
95 \% \mathrm{Cl} \text { ) }\end{array}$ & $1.96(1.91,2.02)$ & $1.80(1.74,1.85)$ & $2.11(2.00,2.23)$ \\
\hline
\end{tabular}

Abbreviation: $\mathrm{Cl}$, confidence interval.

Notes: Data are current as of March 2019 and include diagnosed type 1 and type 2 diabetes combined, but exclude gestationa diabetes. Data from Nova Scotia for individuals aged 1-19 years are excluded; data from Yukon are excluded before 2010/11; data from Nunavut were excluded before 2005/06; data from Saskatchewan are not available for 2016/17. to the start or end of the data and the minimum number of observations between joinpoints were set at four. Agestandardized rates account for differences in age structure of the population over time. We used SAS version 9.3 (SAS Institute Inc., Cary, NC, USA) for all other statistical analysis. Significance was set at $p<.05$.

\section{Results and discussion}

Rates of diabetes are shown in Table 1. In 2016/17, approximately $8.8 \%$ of Canadians ( $9.4 \%$ male, $8.1 \%$ female, aged $\geq 1$ year) were living with diabetes. Diabetes prevalence was higher in adults than in children and youth $(10.9 \%$ vs. $0.3 \%)$. This means that in 2016/17, approximately 3.2 million Canadians were living with diabetes (Figure 1-A); or approximately 1 in 11 adults (aged $\geq 20$ years) and 1 in 333 children and youth (aged 1-19 years). Since 2000/01, age-standardized prevalence rates have increased by an average of $3.3 \%$ per year $(p<.001$; Figure $1-\mathrm{A})$. The greatest increase averaged $5.3 \%$ between 2000/01 and 2006/07 ( $p<.001)$. From $2006 / 07$ to $2010 / 11$, the age-standardized prevalence rate increased an average of $3.3 \%$ per year $(p<.001$ ); from $2010 / 11$ to 2016/17, the age-standardized prevalence rate increased an average of $1.2 \%$ per year $(p<.001)$

In $2016 / 17$, the rate of newly diagnosed diabetes cases was 603.5 per 100000 Canadians (aged $\geq 1$ year). This is approximately 200400 new cases per year, or 
FIGURE 1

Trends in diabetes rates from 2000/01 to 2016/17

\section{A. Number of total cases, and age-standardized prevalence rate of diabetes}

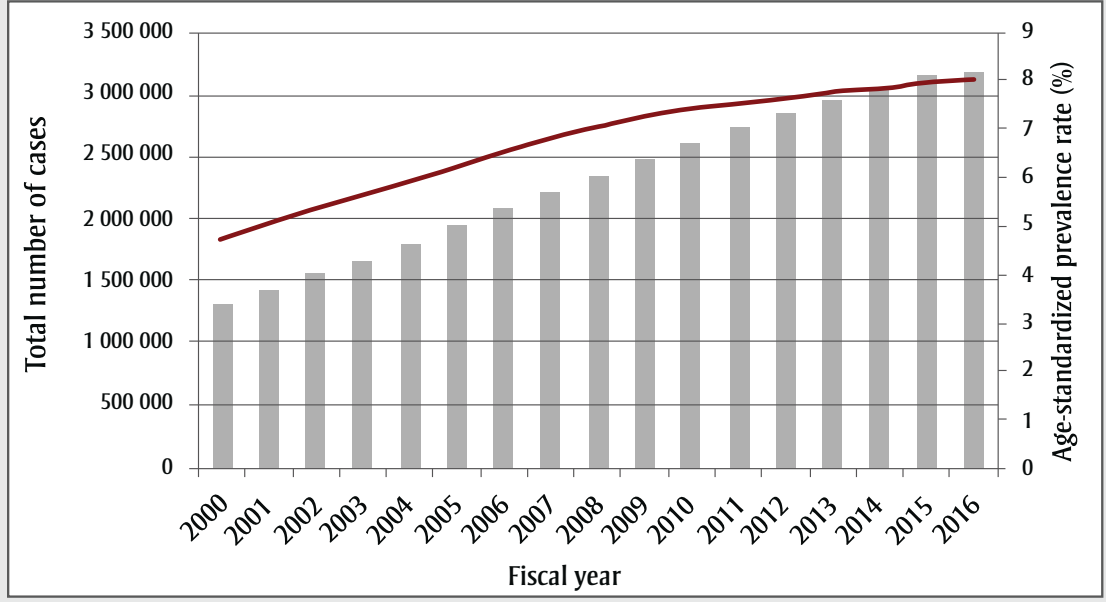

B. Number of new cases, and age-standardized incidence rate of diabetes

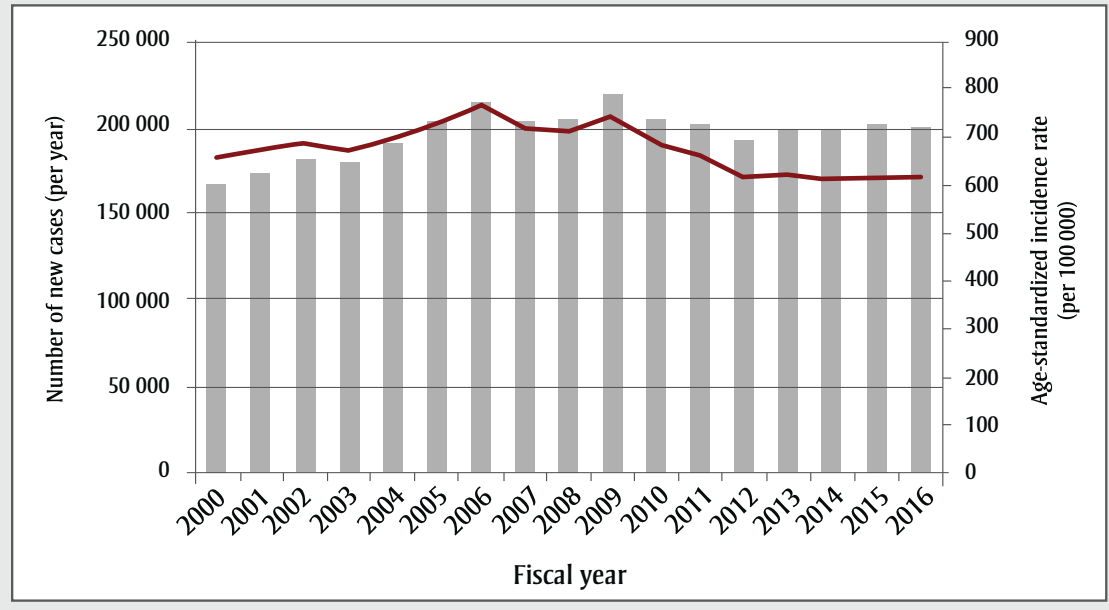

C. Mortality rate and mortality rate ratio of those with diabetes compared to those without diabetes

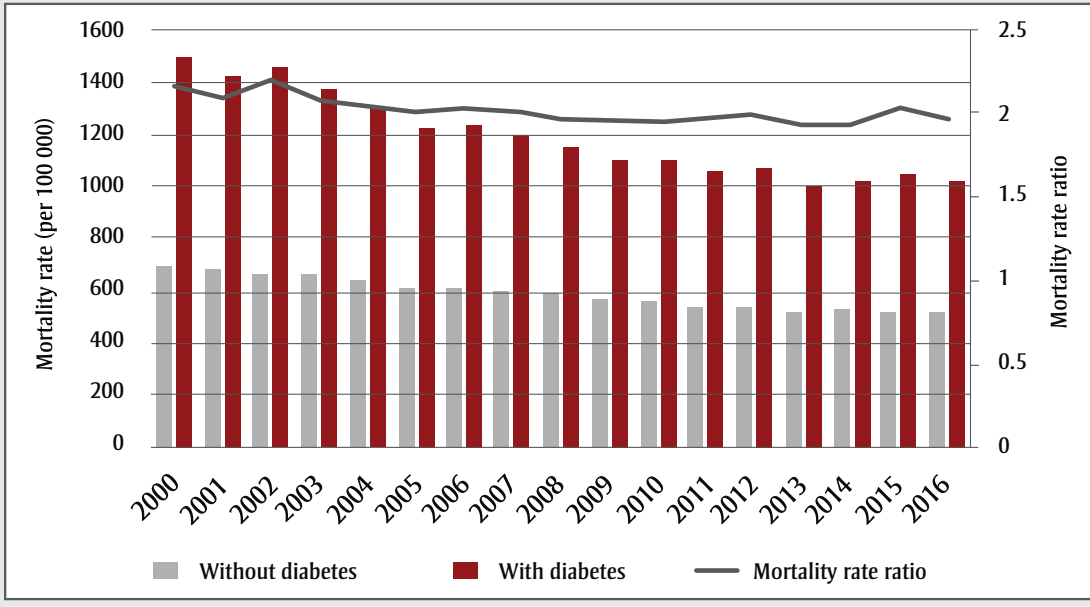

Data source: Canadian Chronic Disease Surveillance System (CCDSS).

Notes: Data are current as of March 2019 and include diagnosed type 1 and type 2 diabetes combined, but exclude gestational diabetes. Data from Nova Scotia for individuals aged 1-19 are excluded; data from Yukon are excluded before 2010/11; data from Nunavut were excluded before 2005/06; data from Saskatchewan are not available for 2016/17.

549 new cases per day (Figure 1-B). From $2000 / 01$ to $2006 / 07$, the age-standardized incidence rate increased by an average of $2.2 \%$ per year $(p<.001)$; from $2006 / 07$ to $2016 / 17$, the age-standardized incidence decreased by an average of $2.2 \%$ per year $(p<.001)$. This means that throughout the surveillance period, agestandardized incidence rates have remained relatively stable (average annual percent change: $-0.5, p=.10$ ). In $2016 / 17$, the all-cause mortality rate for those with diabetes was 1020.6 per 100000 , compared to 519.5 per 100000 for those without diabetes (Figure 1-C). From 2000/01 to 2016/17, there was a decrease in the all-cause mortality rate (average annual percent change: $-2.1, p<.001$ ). This was especially pronounced from $2000 / 01$ to $2013 / 14$, when the all-cause mortality rate decreased by $2.9 \%$ ( $p$ < .001). From 2013/14 to 2016/17 there was no change (annual percent change: $1.7 \%, p=.30)$. Compared to those without diabetes, the mortality rate ratio for those with diabetes is approximately 1.96 (95\% CI: 1.91, 2.02) times higher.

\section{Strengths and limitations}

Compared to other surveillance systems or surveys, the CCDSS provides several advantages. Namely, the CCDSS is able to collect incidence data (more sensitive to changes in the epidemiology of the disease than prevalence); examine trends over time; produce comparable data across provinces and territories (see https:// health-infobase.canada.ca/ccdss/data-tool and search "diabetes" and "geographic comparisons"); and include populationlevel data based on medically diagnosed diseases or conditions. The main limitation of this work is that the current case definition is not able to differentiate between type 1 and type 2 diabetes. The CCDSS is also limited to diagnosed diabetes among health service users. This may underestimate prevalence due to subclinical or undiagnosed diabetes. This work did not examine the influence of other contextual factors that may impact changes in rates over time (e.g. why there were differences in the annual percent change). Future work should continue to refine the case definition for diabetes, including the possibility of including prescription drug databases. ${ }^{14}$ It would also be beneficial to examine contextual factors that may influence rates of diabetes in Canada. Future work could also examine multimorbidity status, as well as calculate costs associated with various diseases. 


\section{Conclusion}

The CCDSS and its unique architecture provide rich data on chronic diseases and conditions in Canada. Over the past 20 years, PHAC, in partnership with all provinces and territories, has been able to report on diabetes rates in Canada. This report provides the most up-to-date information on diabetes prevalence, incidence and mortality in Canada. In 2016/17, $8.8 \%$ of Canadians were living with diabetes. That includes approximately 1 in 11 adults, and 1 in 333 children and youth. Approximately 549 new cases are diagnosed every day. The age-standardized prevalence rate of diabetes has increased over time, whereas the age-standardized incidence rate has remained relatively stable. This may be due, in part, to the fact that people are living longer with the disease, as can be seen in the decline in mortality rate over time. However, mortality risk is much higher among those with diabetes than those without the disease, and diabetes remains one of the major chronic diseases in Canada.

\section{Acknowledgements}

These data were made possible through collaboration between PHAC and the respective provincial and territorial governments of British Columbia, Alberta, Saskatchewan, Manitoba, Ontario, Quebec, New Brunswick, Nova Scotia, Prince Edward Island, Newfoundland and Labrador, Northwest Territories, Yukon and Nunavut. No endorsement by the provinces or territories is intended or should be inferred. Provincial and territorial data were contributed to the CCDSS as of March 2019 (data up to 2016/17).

\section{Conflicts of interest}

The authors declare no conflicts of interest.

\section{Authors' contributions and statement}

AGL conceived of the manuscript and wrote the first draft. AGL and YJG completed the statistical analysis. All authors critically reviewed and provided comments on all aspects of the paper. All authors approved the final version of this work.

The content and views expressed in this article are those of the authors and do not necessarily reflect those of the Government of Canada.

\section{References}

1. Diabetes Canada Clinical Practice Guidelines Expert Committee. Diabetes Canada 2018 clinical practice guidelines for the prevention and management of diabetes in Canada. Can J Diabetes. 2018;42(Suppl 1):S1-S325.

2. Public Health Agency of Canada. Key health inequalities in Canada: a national portrait [Internet]. 2018 May [cited 2018 Nov 21]. Available from: https://www.canada.ca/content /dam/phac-aspc/documents/services /publications/science-research/key -health-inequalities-canada-national -portrait-executive-summary/key_ health_inequalities_full_report-eng .pdf

3. Public Health Agency of Canada (PHAC). Public Health Infobase: Canadian Chronic Disease Surveillance System (CCDSS) [Internet]. [Search "diabetes" under "conditions"]. Ottawa (ON): PHAC [updated 2019 Mar 14; cited 2018 July 17]. Available from: https://infobase.phac-aspc.gc.ca /ccdss-scsmc/data-tool/

4. Statistics Canada. Health Fact SheetsDiabetes, 2017. Ottawa (ON): Statistics Canada; 2018 [Catalogue No. 82-625X].

5. Health Canada. Responding to the challenge of diabetes in Canada: first report of the National Diabetes Surveillance System (NDSS). Ottawa (ON): Government of Canada; 2003. [Catalogue No. H39-4/21-2003E]. 122 p.

6. Public Health Agency of Canada (PHAC). The Canadian Chronic Disease Surveillance System - an overview. Ottawa (ON): PHAC [updated 2018 Oct 12; cited 2019 July 10]. Available from: https://www.canada.ca/en /public-health/services/publications /canadian-chronic-disease-surveillance -system-factsheet.html

7. CCDI Steering Committee, Public Health Agency of Canada. How healthy are Canadians? A brief update. Health Promot Chronic Dis Prev Can. 2018; 38(10):385-7. Erratum in Health Promot Chronic Dis Prev Can. 2019;39(2):63.

8. Hamm NC, Pelletier L, Ellison J, et al. Trends in chronic disease incidence rates from the Canadian Chronic Disease Surveillance System. Health Promot Chronic Dis Prev Can. 2019; 39(6/7):216-24.
9. Public Health Agency of Canada. How healthy are Canadians? Ottawa (ON): Government of Canada. 2016 [Catalogue No: HP40-167/2016E]. 46 p.

10. Feely A, Lix L, Reimer K. Estimating multimorbidity prevalence with the Canadian Chronic Disease Surveillance System. Health Promot Chronic Dis Prev Can. 2017;37(7):215-22.

11. Lix L, Ayles J, Bartholomew S, et al. The Canadian Chronic Disease Surveillance System: a model for collaborative surveillance. Int J Pop Data Sci [Internet]. 2018;3(3). Available from: https://ijpds.org/article/view/433

12. Lix L, Reimer K. The Canadian Chronic Disease Surveillance System: a distributed surveillance model. Online J Public Health Inform [Internet]. 2017;9(1):e140. doi: 10.5210/ojphi .v9i1.7726.

13. James RC, Blanchard JF, Campbell D, et al. A model for non-communicable disease surveillance in Canada: the Prairie Pilot Diabetes Surveillance system. Chronic Dis Can. 2004;25(1): 7-12.

14. Liscombe LL, Hwee J, Webster L, et al. Identifying diabetes cases from administrative data: a populationbased validation study. BMC Health Service Research [Internet]. 2018; 18(1):316 